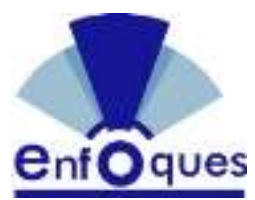

\section{EJECUCIÓN DE PROYECTOS DE VIVIENDAS DE INTERÉS SOCIAL DEL SECTOR CONSTRUCCIÓN}

\author{
MANAGEMENT OF PROJECTS EJECUTION PHASE OF SOCIAL \\ HOUSING CONSTRUCTION SECTOR
}

\author{
Elizabeth Dubuc
}

Artículo recibido julio 2019 | Arbitrado agosto - septiembre 2019 | Publicado 01 de octubre 2019

\section{Resumen}

El artículo a continuación describe la fase de ejecución de proyectos de viviendas de interés social del sector construcción en una ciudad de Venezuela, sustentándose teóricamente en los aportes de Cartay, PMBOK, y Chamoun, entre otros. La metodología fue de tipo descriptiva, con diseño no experimental, transeccional de campo. La técnica de recolección de información se basó en la observación mediante encuesta, utilizando como instrumento un cuestionario contentivo de 65 items. La validez se obtuvo a través de 5 jueces de expertos, y un coeficiente de confiabilidad 0.975, medido a través del alpha Cronbach, el cual fue aplicado a una población conformada por un total de 15 personas, constituida ésta por 5 gerentes de proyectos del área de construcción y 10 personas de otros departamentos que intervienen en la elaboración y ejecución de proyectos civiles. Para el análisis de los resultados se utilizó la estadística descriptiva con tablas de frecuencias absolutas y porcentuales. Los resultados del estudio evidencian debilidades mayormente por falta de definición de los alcances del proyecto, así como deficiencias en el manejo de las diferentes actividades de la fase de ejecución.

Palabras clave: Modelo gerencial; fase de ejecución; viviendas de interés social

\begin{abstract}
The article below describes the execution phase of social interest housing projects in the construction sector in a city in Venezuela, theoretically based on the contributions of Cartay, PMBOK, and Chamoun, among others. The methodology was descriptive, with a non-experimental, transectional field design. The information gathering technique was based on observation by means of a survey, using as a tool a 65 -item content questionnaire. The validity was obtained through 5 judges of experts, and a reliability coefficient 0.975 , measured through the Cronbach alpha, which was applied to a population made up of a total of 15 people, constituted by 5 project managers in the area of construction and 10 people from other departments involved in the development and execution of civil projects. For the analysis of the results, descriptive statistics were used with tables of absolute and percentage frequencies. The results of the study show weaknesses mainly due to lack of definition of the scope of the project, as well as deficiencies in the management of the different activities of the execution phase.
\end{abstract}

Key words: Management model; execution phase; social interest housing
Elizabeth Dubuc

dubucelizabeth62@gmail.com Universidad Rafael Belloso Chacín, Venezuela

Ingeniero Civil, Magister en Gerencia de Proyecto Industriales de la Universidad Rafael Belloso Chacín. 


\section{INTRODUCCIÓN}

Unos de los graves problemas que confronta la humanidad es el déficit de viviendas. Todos los países del mundo, en mayor o menor grado, sufren las consecuencias de tan grave situación.

En América latina, países, como Colombia, Chile, Ecuador, Perú y el Caribe buscan constantemente modelos que les permita mejoras a las condiciones de vida de la población de bajo recursos y de esa manera mejorar la calidad de vida de la población. Los gobiernos locales, academia, y comunidades se asocian entre ellos de forma independiente para proyectar programas de desarrollo urbano. Sin embargo, la deficiencia sigue presente.

En Venezuela esta problemática es característica, aqueja a un número de venezolanos y en algunos casos, varias generaciones de familiares viven bajo un mismo techo o en improvisadas construcciones en terrenos vulnerables. Hay que resaltar los factores que inciden en esta problemática los cuales tienden a ser administrativos, legales, técnicos, comerciales, financieros entre otros. Por lo general este tipo de proyectos de viviendas de interés social en su fase de ejecución se ve cuestionada en cuanto a materiales de construcción, falta de experiencia de planificadores y ejecutores, retrasos en la procura y equipos, problemas de personal, presupuestos no ajustados a la realidad, pobre definición de los alcances del proyecto, pago a personal que no efectúa sus labores, interrupción de obras, entre otros.

Muy a pesar de los programas habitacionales implementados por diversos organismo regionales como nacionales, entre ellos Corpozulia, PDVSA, Metro de Maracaibo en conjunto con La
Gran Misión Vivienda, en el Estado Zulia, esta problemática es similar; igualmente se dan casos de incumplimiento de las metas de construcción establecidas por el propio gobierno regional, no se cumple por diversos factores ya sea por desorganización de los recursos asignados a nivel institucional o entre otros, por burocratización en los procesos de gestión entre el ente contratante y la empresa contratada.

Lo anteriormente señalado, es común entre las empresas constructoras y entes contratantes, encargados de la ejecución de proyectos de viviendas de interés social, sin embargo, hay empresas en este tipo de proyectos que obtienen resultados exitosos como es el caso de las empresas tecnoconsult, e IraniHousing entre otras. Estas son organizaciones que trabajan de manera conjunta para alcanzar un objetivo en un tiempo determinado. Es decir, sus resultados indican que trabajan con un proceso de gestión bien implementado.

En este sentido, Cartay, (2010), define proyecto como un conjunto ordenado de acciones que tienden a la realización de un determinado fin. Sea sencillo o complejo, todo proyecto tiene un inicio $y$ un fin definidos en el tiempo, y se concibe como una secuencia de actividades tendentes a buscar, analizar y coordinar un conjunto de informaciones y datos que justifiquen, según ciertos criterios, su ejecución.

Actualmente, los proyectos son mecanismos utilizados en las empresas para implementar los requerimientos necesarios que permiten llegar a la meta propuesta en un tiempo determinado. Se debe tener en cuenta que gerenciar un proyecto compromete con la resolución y ejecución de tareas planificadas que no pueden ser manejadas eficientemente ni en el menor 
tiempo, mediante estructuras organizativas tradicionales.

Para Cartay (2010), gerencia es la planificación, organización, dirección y control de los recursos asignados a un proyecto que debe ser completado para alcanzar metas y objetivos específicos. Es así como, emprender un proyecto de construcción habitacional, cualquiera que este sea, precisa asumir tareas y actitudes gerenciales a fin de lograr el éxito mediante el alcance de los objetivos definidos en el proyecto.

Es de resaltar, en el caso de estudio, que las empresas contratistas seleccionadas poseen un sistema gerencial diseñado con el propósito de cumplir con la metas planteadas y a pesar de existir una estandarización de actividades, no aplican criterios de comparación, reales versus lo planificado, a los resultados lo que evidencia dificultades en la ejecución y en el seguimiento físico-financiero.

Las limitaciones antes descritas confirman la necesidad de mejora del desempeño de las empresas contratistas dedicadas a la construcción de viviendas de bajo costo, para lo cual es necesaria la incorporación de gerentes comprometidos que conozcan y apliquen un modelo de gerencia eficaz y eficiente con el cual enfrentar la gran variedad de problemas y situaciones que requieren desarrollar nuevas destrezas y habilidades gerenciales para aportar soluciones.

Sobre todo es necesaria la adaptación a los cambios constantes que surgen en este tipo de proyectos, lo que incluye saber integrar su equipo de trabajo, saber aplicar controles de seguimiento a su proyecto, ser un buen líder, saber cómo tomar decisiones cuando el proyecto así lo requiera, identificar y resolver obstáculos a tiempo y de manera eficiente. Todo lo anteriormente señalado garantiza a la empresa optimar la administración de cualquier proyecto civil.

De lo antes descrito, surge la inquietud de conocer ¿Cómo es la ejecución de proyectos de viviendas de interés social del sector construcción en el Estado Zulia?

\section{Fundamentación teórica}

Como parte medular del desarrollo de las bases teóricas, es necesario conceptualizar la variable en estudio, así como los elementos que la componen a fin de entender y reconocer la importancia de la misma.

En este sentido, Chamoun, (2007) define gerencia como la aplicación de conocimientos habilidades, técnicas y herramientas a las actividades del proyecto con el fin de superar las expectativas de los involucrados.

De igual manera Krygier (2010), define gerencia como un cuerpo de conocimientos aplicables a la dirección efectiva de una organización. En la actualidad existe un consenso, entre muchos autores, al señalar que el término gerencia se puede definir como un proceso que implica la coordinación de todos los recursos disponibles en una organización: humanos, físicos, tecnológicos y financieros, entre otros.

De esta manera, se pueden distinguir tres (3) aspectos claves para definir la gerencia como proceso: coordinación de recursos de la organización, ejecución de funciones gerenciales o también llamadas administrativas, como medio de lograr la coordinación, y establecimiento del propósito del proceso gerencial, es decir, 
donde se quiere llegar o que es lo que se desea lograr.

Según, Cartay (2010), la gerencia de proyectos puede ser descrita como la planificación, organización, dirección, y control de los recursos asignados a un proyecto que debe ser completado para alcanzar metas y objetivos específicos.

La gerencia de proyectos permite acometer tareas que no pueden ser manejadas eficientemente mediante estructuras organizativas tradicionales, por lo que se le considera como un desarrollo fundamental en la búsqueda de nuevas formas de organizaciones futuras, diseñadas para integrar esfuerzos complejos $y$ disminuir la burocracia. Igualmente la gerencia de proyectos permite acometer la ejecución de actividades con un mínimo de interrupción para el funcionamiento normal de los negocios.

Según el análisis los dos primeros autores difieren en sus enfoques dirigidos más hacia la estructura organizativa, tomando posición de Cartay (2010), la gerencia de proyecto, se refiere a la administración del proyecto desde su inicio hasta su culminación. De esta manera debe encargarse de planificar coordinar y llevar seguimiento de todo lo relacionado con calidad, avance y costos en cada una de las actividades que conforman el proyecto, de manera de satisfacer las necesidades del cliente o empresa contratante. De acuerdo a estas definiciones, la gerencia es un tipo de administración cuyo rasgo particular es el énfasis en la búsqueda de la eficiencia, es decir, los proceso administrativos deben transformarse, tomar características que permitan no solo obtener los fines, sino también resultados económicos favorables.

En este orden de ideas, el PMBOK, (2008) define proyecto como un esfuerzo temporal que se lleva a cabo para crear un producto servicio, o resultado único. Quiere decir que el producto o servicio es diferente de alguna manera distintiva de todos los proyectos o servicios similares.

Asimismo, Cartay, (2010), define proyecto como el conjunto ordenado de acciones que tienden a la realización de un determinado fin. Sea sencillo o complejo, todo proyecto tiene un inicio $y$ un fin definido en el tiempo y se concibe como una secuencia de actividades tendientes a buscar, analizar y coordinar un conjunto de informaciones y datos que justifiquen, según ciertos criterios de ejecución.

De lo mencionado se puede concluir que proyecto es el conjunto ordenado de procedimientos con un fin en un tiempo determinado. La definición de proyectos para el PMBOK (2008), se refiere a un esfuerzo temporal para crear productos o servicios es decir, más hacia el área de mercadeo o plan de negocios. Tomando la posición de Cartay (2010), hay muchos tipos de proyectos desde los complejos hasta los más sencillos, pero todos parten de la misma base que es el conjunto de acciones relacionadas entre sí.

Según Pmbok (2013), el ciclo de vida de un proyecto se define en función de las diferentes fases, generalmente secuenciales y en ocasiones superpuestas, que atraviesa hasta llegar a su culminación, así se pueden mencionar como fases de un proyecto: fase de inicio, de planificación, de ejecución y de cierre; en cada una de las cuales se dan actividades propias de la fase, las cuales están al mismo tiempo relacionadas todas entre sí.

En el caso específico de esta investigación el objeto de estudio es describir la fase de ejecución de los proyectos de construcción de viviendas de 
interés social en el Estado Zulia, con la finalidad de conocer la condición actual de las empresas en estudio durante el desarrollo de la mencionada fase.

En este sentido el PMBOK (2003) refiere que dirigir la ejecución de un proyecto es ejecutar las actividades definidas en el plan de trabajo del proyecto para alcanzar los objetivos del mismo. Estas actividades son variadas e incluyen recopilar y documentar las lecciones aprendidas e implementar las actividades aprobadas de mejora del proceso.

EI PMBOK (2013), expone que la fase de ejecución es compleja ya que se deben considerar una gran cantidad de factores y es en ella cuando se promueve la realización de actividades que conllevan al cumplimiento de los objetivos, ya sea al normalizar los procesos o mediante la aplicación, durante su desarrollo, del análisis, documentación y planificación de soluciones o la realización de actividades que sirvan de solución a posibles cambios en el alcance del proyecto.

Es así como, para el PMBOK (2013) la fase de ejecución consiste no sólo en cumplir con el cronograma de trabajo, sino que también considera la administración del recurso humano, financiero, técnico y tecnológico. En cuanto al aseguramiento de la calidad que también incluye esta fase, el PMBOK (2013) destaca que en la fase de ejecución se necesita un proceso que asegure la calidad mediante auditorías de los requisitos de la misma y de los resultados obtenidos a partir de medidas de control, para garantizar así la utilización de definiciones operacionales y normas acordes que permiten evaluar la validez de peticiones de modificaciones, en la planificación realizada.

En cuanto al alcance del proyecto, el PMBOK (2008), lo define como el enunciado que contempla todos los procesos necesarios para asegurar que el proyecto incluya la totalidad del trabajo requerido, a fin de completar el proyecto satisfactoriamente, es decir, describe en detalle, los productos entregables del proyecto y el trabajo necesario para crear tales productos entregables. El enunciado del alcance de proyecto también proporciona un entendimiento común del alcance del proyecto entre los interesados del proyecto, y describe los principales objetivos del mismo.

El alcance del proyecto permite al equipo del proyecto realizar una planificación más detallada, guía el trabajo del equipo de proyecto durante la ejecución y proporciona la línea base para evaluar si las solicitudes de cambio o trabajo adicional están comprendidas dentro o fuera de los límites del proyecto.

Es así como el grado y nivel de detalle del enunciado del alcance del proyecto define el trabajo a realizar y cual quedará excluido lo que puede determinar el nivel de éxito con que el equipo de dirección del proyecto planifique, gestione y controle la ejecución del proyecto. Según el PMBOK (2008) el detalle en el nivel del enunciado del alcance de proyecto incluye los objetivos del proyecto, que a su vez incluyen los criterios medibles de éxito del proyecto. Los proyectos pueden tener una amplia variedad de objetivos de negocio, de costos, de cronograma, técnicos y de calidad.

Asimismo, la descripción del alcance de producto, describe la característica del producto, servicio o resultado para el cual se creó el proyecto. Generalmente estas características serán menos detalladas en las fases iniciales y más detalladas en las fases posteriores, a medida que las características del producto se van elaborando gradualmente. Si bien la forma y el contenido de las características pueden 
variar, la descripción del alcance deberá ser siempre lo suficientemente detallada como para que sirva de apoyo a la planificación posterior del alcance de proyecto. Es así como se deben describir los requisitos, las restricciones y los límites del proyecto para tener claridad en cuanto a las condiciones que se deben cumplir, las capacidades de los entregables para satisfacer un contrato, norma, especificación o cualquier otro documento formalmente impuesto; así como conocer qué incluye o qué está excluido del proyecto y cuáles son las restricciones específicas del mismo que limitan las opciones del equipo del proyecto.

Según, Miranda (2005), en el alcance de proyecto se debe estipular en forma clara e inequívoca cada una de las obras que forman parte del contrato, e indicar el compromiso del contratista en cada una de ellas, con base a los contenidos del pliego de licitación y la oferta correspondiente. Es decir, se puede definir al alcance del proyecto como una guía primordial para el inicio de un proyecto, por lo que éste debe estar bien definido en la primera etapa del proyecto, lo que evita inconvenientes que generan pérdidas económicas durante el desarrollo del proyecto.

En función de las apreciaciones de los autores antes mencionados se infiere que el alcance de un proyecto implica un proceso con diferentes actividades que deben ser tomadas en cuenta en forma detallada desde la primera fase del proyecto y continuar de la misma manera durante su desarrollo, ya que al estar bien definido el alcance se evitan dificultades en su recorrido por las diferentes fases del proyecto.

En este mismo orden de ideas, Briceño (2006), expone que la programación de actividades, se inicia con un plan maestro, cuyas estimaciones iníciales son precisadas cada vez con mayor detalle por la programación cuantitativa, a medida que progresa la ingeniería. El programa de la construcción es integrado, evidentemente, con el programa general del proyecto, y en su formulación están contenidas las programaciones parciales de detalle, de cada uno de los subcontratos o paquetes, en los cuales esta modulada la obra.

De igual manera expresa el mencionado autor que la programación de actividades de obras de construcción, depende de factores como volumen, especialización y diversidad de disciplinas a desarrollar, numero de subproyectos, interrelaciones de los equipos de trabajo, consideraciones espaciales con relación a las instalaciones existentes, comprensión de la programación y otros.

Según Guido y Clements, (2003), definición de actividades, es una relación de actividades específicas, detalladas, necesarias para realizar el proyecto global, en particular en proyectos pequeños. Sin embargo, para aquellos proyectos en los que se usa una estructura de división de trabajo, la persona o equipo responsable de cada tarea puede definir las actividades individuales.

En este mismo orden PMBOK, (2008), expresa que definir las actividades del cronograma implica identificar y documentar el trabajo que se planifica realizar, lo cual comprende identificar los productos entregables al nivel más bajo de la estructura de desglose del trabajo (EDT) para convertirlos en paquetes de trabajo. Los paquetes de trabajo del proyecto se planifican descompuestos en componentes más pequeños denominados actividades del cronograma, para proporcionar una base con la cual estimar, establecer el cronograma, ejecutar, supervisar y controlar el trabajo del proyecto.

En relación con la programación de actividades el PMBOK (2008), describe el método de pert cpm como uno de los 
métodos en programación de actividades, mientras que Briceño (2006), y Guido y Clements (2003), se refieren a un conjunto de actividades que nos ayuda a prever cómo lograr los objetivos de un proyecto, descrito de una manera más sencillo y menos técnico.

Sin embargo, la posición del PMBOK (2008), se refiere a la programación de actividades con una herramienta gerencial que permite crear un plan de trabajo de diferentes niveles para controlar y guiar los objetivos señalados durante el proyecto, lo cual orienta el proceso, que incluye la planificación en costo, materiales, alcance, el control, cierre de la obra, productos entregables y salidas; aplica los diferentes métodos de programación de actividades, la cual ayuda a visualizar todo el proyecto.

En relación con la procura, Cartay, (2010), sugiere una agrupación de funciones existentes que tenga relación con el abastecimiento de materiales para garantizar la operacionalidad del proyecto. En este sentido el sistema de materiales tiene por objetivo abastecer a los diferentes sectores del proyecto, de materiales necesario para la ejecución para satisfacer así los requisitos cuantitativos de costo y de oportunidad.

De igual manera, el PMBOK (2008), se refiere a la procura en los proyectos, como el proceso que se lleva a cabo fuera del equipo de proyecto, para comprar o adquirir los productos, servicios o resultados necesarios para realizar el trabajo. Para lo cual se debe tomar una serie de consideraciones a la hora de gestionar la procura como es: planificar las compras y adquisiciones, planificar la contratación, documentar los requisitos, de los productos o servicios y resultados, identificar los vendedores, solicitar respuesta de vendedores, obtener información de presupuesto, licitaciones, ofertas o propuestas, según corresponda.
Selección de vendedor, revisar ofertas, elegir entre posibles vendedores y negociar un contrato por escrito por cada vendedor, Administración de contrato, gestionar el contrato y la relación entre el comprador y el vendedor, revisar y documentar cual es o fue el rendimiento del vendedor, a fin de establecer las acciones correctivas y proporcionar una base para relaciones futuras.

Cierre de contrato, completar y aprobar cada contrato, incluida la resolución de cualquier tema abierto y cerrar cada contrato aplicable al proyecto o una fase del proyecto.

Para el primer autor mencionado, procura es un recurso necesario dentro de un proyecto como equipos, materiales y herramientas, mientras el PMBOK (2008), es un proceso de gestión de adquisición y materiales, que explica de manera detallada todo los procesos de gestión, que incluye la actividad procura desde su planificación hasta su cierre de contrato, lo cual constituye una excelente base para programar actividades para este tipo actividad y para manejar los proveedores y compras necesarias y requeridas en el desarrollo del proyecto.

En cuanto al manejo de contrato, Rico,(2009), define administración del proyecto o manejo del contrato, como la coordinación de un grupo de actividades, donde el administrador planea, organiza, dirige y controla para lograr el objetivo dentro de restricciones de tiempo, costo, desempeño y funcionamiento del proyecto final, es administrar el cambio.

Asimismo, según el PMBOK (2008), la administración del contrato se define como, el proceso de gestionar la relación entre comprador y vendedor, revisar y documentar cual es o fue el rendimiento de un vendedor a fin de establecer acciones correctivas necesarias 
y proporcionar una base para las relaciones futuras con el vendedor, gestionar cambios relacionados con el contrato, cuando corresponda, y gestionar la relación contractual con el comprador externo del proyecto. De los dos autores mencionados, el primero describe manejo de contrato como un grupo de actividades que el gerente $o$ líder del proyecto debe conocer e implementar a lo largo del proyecto, en cambio el PMBOK (2008), lo maneja como un proceso de gestión de todas las etapas de un proyecto. La posición del primer autor permite inferir que el manejo de contrato se refiere, a como los lideres o gerentes deben aplicar una serie de técnicas gerenciales en costo, tiempo y calidad en un tiempo determinado.

Con referencia a la inspección de la obra Merrit, Loftin, Rickett (2004), definen que la inspección y consulta periódica durante la construcción, son normalmente parte de las responsabilidades del ingeniero inspector. Estas labores comprenden visitas periódicas al lugar de la construcción. La función del ingeniero del lugar de construcción, y del personal es inspeccionar que el trabajo se apegue a los requisitos del contrato, a los documentos y conceptos del diseño.

La inspección de obra, se refiere a la revisión periódica, de cómo van los trabajos de ejecución del proyecto, es ir al sitio y comprobar si todo realmente va según los cronogramas realizados.

Según el autor la inspección de obra, no solo es revisión que corresponde al ingeniero inspector, sino que ésta incluye inspecciones tipo visitas periódicas, no solo del ingeniero inspector, sino también del ingeniero constructor o líder del proyecto y del personal que de alguna manera deba llevar seguimiento y control de la obra.

En relación con la forma de pago, Briceño (2006), expresa que las formas de pago establecidas en los contratos de obras son básicamente tres: Contrato Por Suma Alzada: El cual establece un monto global, fijo, por las obras a realizar, atendido el alcance de las mismas.

La aplicación de esta modalidad requiere haber desarrollado la ingeniería completa del proyecto o subproyecto especifico, única forma de conocer con exactitud sus especificaciones, obras físicas, listados de equipos, ubicaciones de suministros y recursos involucrados en la ejecución. Sin información a este nivel de detalle, la empresa constructora es incapaz de presentar un presupuesto técnicamente sustentado, y el cliente no tiene herramientas para evaluarlo.

La segunda modalidad de pago es la de control por administración delegada o administración directa la que establece el pago de un precio igual a los costos (estimados), más honorarios de administración (gastos, supervisión general, utilidad y otros), básicamente precio es igual a presupuesto estimado más honorarios.

La tercera forma de pago es la de contrato por precios unitarios que según Briceño (2006), es una fórmula contractual donde el precio de la obra resulta básicamente de multiplicar la cantidad de obra por su precio unitario.

Usualmente, la cantidad física de la obra encargada no es conocida con exactitud, por lo que es el usuario quien asume la responsabilidad de controlar esta variable y los aumentos imprevistos que ocurran. Por otra parte, los precios unitarios convenidos (validos dentro de cierto rango de unidades físicas) caen exclusivamente dentro del ámbito de control del contratista.

La aplicación del contrato de precios unitarios necesita de precisiones muy rigurosas de las características técnicas de los respectivos trabajos, y exactitud de 
medición de la variable cantidad durante la ejecución, HH horas hombres (ingeniero, dibujante, supervisor, técnico), M3 (excavación, rellenos, fundaciones), ML (cableado, pasamanos, piping, cercos), unidades (instalaciones de motores tableros, luminarias, bandejas).

En este caso un componente de costo indirecto (provisión para equipos, gastos de instalación de faenas, gastos generales y utilidad), el que se asumió fijo (suma alzada) dentro de cierto rango de cantidad de obra. Si la cantidad de obra aumenta al doble y algo similar ocurre con los plazos normales de ejecución, el contratista, sin duda, plantea renegociar su asignación de costos indirectos

Finalmente según Guido y Clements, (2003) el contrato es considerado como un medio para establecer buenas comunicaciones entre el cliente y el contratista lo que les permite arribar a una comprensión mutua con claras expectativas que aseguran el éxito del proyecto.

EI PMBOK (2008), define el proceso de cierre de contrato como un respaldo al cierre del proyecto, ya que incluye la verificación de que todo el trabajo y todos los productos entregables han sido aceptados.

El proceso del cierre de contrato también incluye actividades administrativas, como por ejemplo actualización de registros para reflejar los resultados finales y archivo de dicha información para su uso en el futuro. El cierre del contrato aborda a cada contrato aplicable al proyecto o a una fase del proyecto. En proyectos de múltiples fases, el plazo de un contrato puede ser aplicable solo a una fase determinada del proyecto.

En estos casos, el proceso de cierre de contrato solo cierra los contratos aplicables sólo a esa fase del proyecto. Las reclamaciones pueden quedar sujetas a litigio después del cierre del contrato. Los términos y condiciones del contrato pueden prescribir procedimientos específicos para el cierre del contrato.

La finalización anticipada de un contrato es un caso especial de cierre de contrato, y puede resultar de un acuerdo mutuo entre las partes o del incumplimiento de una de las partes. Los derechos y responsabilidades de las partes, en caso de finalización anticipada, están incluidos en una cláusula de finalización de contrato. Basándose en esos términos y condiciones del contrato, el comprador puede tener derecho a dar por finalizada la totalidad del contrato o una parte del proyecto, por justa causa o conveniencia, en cualquier momento.

Sin embargo, de acuerdo con dichos términos y condiciones del contrato, es posible que el comprador tenga que compensar al vendedor por los preparativos y por todo el trabajo completado y aceptado relacionado con la parte del contrato que se da por finalizada.

Según, Briceño (2006), el cierre del proyecto involucra el ordenamiento $y$ documentación de todas aquellas actividades que deben traspasarse a la organización permanente, lo cual trasciende más allá del periodo pre operacional. Su delimitación temporal, por lo tanto, es incierta y se superpone habitualmente con los procedimientos de la puesta en marcha.

Para el PMBOK (2008) el cierre de proyecto se refiere a cada fase de los diferentes procesos que incluye la gestión del proyecto, en cambio para Briceño, (2006), incluye la documentación de todo lo que implica el cierre del contrato al finalizar la puesta en marcha. 
En función de lo expresado por los autores se considera el cierre del contrato, como la última etapa de la fase de ejecución de un proyecto, es el momento en el cual se debe realizar un expediente, que incluya todos los documentos necesarios para el cierre, en ese expediente debe reposar desde el acta de inicio de la obra hasta la fianza y recepción definitiva de la obra, con el objetivo de evitar futuros inconvenientes y al mismo tiempo utilizar esta información como base para futuros proyectos similares.

\section{MÉTODO}

Tomando en consideración el tipo de planteamiento del problema y los objetivos a alcanzar se puede decir que esta investigación fue de tipo descriptiva, con un diseño de campo transeccional. La población de estudio estuvo conformada por un total de 15 personas, constituida ésta por 5 gerentes de proyectos del área de construcción y 10 personas de otros departamentos que intervienen en la elaboración y ejecución de proyectos civiles.

Para la recolección de los datos se utilizó la técnica de la encuesta y como instrumento un cuestionario contentivo de 65 items. La validez de dicho instrumento se obtuvo a través de 5 jueces de expertos, y su coeficiente de confiabilidad se obtuvo a través del alpha Cronbach, con resultado de 0.975 , lo cual indica una alta confiabilidad.

Para el análisis de los resultados se utilizó la estadística descriptiva con tablas de frecuencias absolutas y porcentuales. De igual manera para la constatación e interpretación de los niveles y puntajes para los indicadores y dimensión de la variable, se aplicó el baremo que muestra la tabla 1. Utilizando la escala de 1-4 con alternativas de cuatro (4) respuestas.

Tabla 1. Baremo ponderado para la categorización de los estadísticos descriptivos de las variables

\begin{tabular}{clcc}
\hline No & Alternativas & Rango & Categoría \\
\hline 4 & siempre & $3.26-4$ & Alto \\
3 & casi siempre & $2.51-3.25$ & Moderado \\
2 & algunas veces & $1.76-2.50$ & Bajo \\
1 & iunca & $1-1.75$ & Muy Bajo \\
\hline
\end{tabular}

\section{RESULTADOS Y DISCUSIÓN}

Una vez aplicado el cuestionario a la población objeto de estudio de las empresas contratistas, se obtuvo la información, se tabularon los datos y se muestran en tabla 2 los resultados estadísticos, expresados en un lenguaje formal y sencillo que permitió su interpretación. 
Tabla 2. Distribución Frecuencial de la fase de ejecución de proyectos

\begin{tabular}{|c|c|c|c|c|c|c|c|c|c|c|c|c|c|c|}
\hline \multirow[t]{2}{*}{ Alternativas } & \multicolumn{2}{|c|}{ Alcance. } & \multicolumn{2}{|c|}{$\begin{array}{l}\text { Programación } \\
\text { Actividades. }\end{array}$} & \multicolumn{2}{|c|}{ Procura. } & \multicolumn{2}{|c|}{$\begin{array}{c}\text { Manejo de } \\
\text { Contrato }\end{array}$} & \multicolumn{2}{|c|}{$\begin{array}{l}\text { Inspección } \\
\text { de Obra. }\end{array}$} & \multicolumn{2}{|c|}{$\begin{array}{c}\text { Forma de } \\
\text { Pago. }\end{array}$} & \multicolumn{2}{|c|}{$\begin{array}{l}\text { Cierre de } \\
\text { Contrato. }\end{array}$} \\
\hline & $\mathrm{fa}$ & $\%$ & $\mathrm{fa}$ & $\%$ & fa & $\%$ & $\mathrm{fa}$ & $\%$ & $\mathrm{fa}$ & $\%$ & fa & $\%$ & fa & $\%$ \\
\hline (4) Siempre & 0 & 0 & 1 & 6,7 & 0 & 0 & 3 & 20 & 0 & 0 & 6 & 40 & 0 & 0 \\
\hline (3) Casisiempre & 4 & 26,7 & 5 & 33,3 & 5 & 33,3 & 6 & 40 & 5 & 33,3 & 6 & 40 & 5 & 33,3 \\
\hline (2) Algunas Veces & 5 & 33,3 & 2 & 13,3 & 4 & 26,7 & 5 & 33,3 & 4 & 26,7 & 2 & 13,3 & 4 & 26,7 \\
\hline (1) Nunca & 6 & 40 & 7 & 46,7 & 6 & 40 & 1 & 6,7 & 6 & 40 & 1 & 6,7 & 6 & 40 \\
\hline TOTAL & 15 & 100 & 15 & 100 & 15 & 100 & 15 & 100 & 15 & 100 & 15 & 100 & 15 & 100 \\
\hline XIndic ador & \multicolumn{2}{|c|}{2,10} & \multicolumn{2}{|c|}{2,13} & \multicolumn{2}{|c|}{2,07} & \multicolumn{2}{|c|}{2,72} & \multicolumn{2}{|c|}{2,07} & \multicolumn{2}{|c|}{2,91} & \multicolumn{2}{|c|}{2,07} \\
\hline Categoria IND & \multicolumn{2}{|c|}{ Bajo } & \multicolumn{2}{|c|}{ Bajo } & \multicolumn{2}{|c|}{ Bajo } & \multicolumn{2}{|c|}{ Moderado } & \multicolumn{2}{|c|}{ Bajo } & \multicolumn{2}{|c|}{ Moderado } & \multicolumn{2}{|c|}{ Bajo } \\
\hline X Dimensión & \multicolumn{14}{|c|}{2,29} \\
\hline Categoria DIM & \multicolumn{14}{|c|}{ Bajo } \\
\hline
\end{tabular}

La tabla 2, muestra el comportamiento de la fase de ejecución de proyectos que al analizarse puede apreciarse que para el alcance, el mayor porcentaje lo obtuvo la alternativa de respuesta nunca con un $40 \%$, seguido de algunas veces con un 33.3\%, luego la alternativa casi siempre que alcanzó un $26.7 \%$ y por último la alternativa siempre que no presentó puntuación alguna.

Con respecto a la media del alcance, este dio como resultado 2.10, lo que comparado con el baremo de interpretación lo ubica en la categoría de bajo. Este valor refleja la presencia, en la fase de ejecución, de ciertas deficiencias como falta de definición y detalle en el enunciado de los proyectos de viviendas de interés social.

Este resultado difiere del planteamiento de Miranda (2005) quien, define como alcance de proyecto la descripción detallada, de los trabajos a ejecutar, la cual se convierte en guía para el contratista a la hora de proceder con la ejecución de dicha obra o proyecto.

En cuanto a la programación de actividades, el mayor porcentaje lo obtuvo la alternativa de respuesta nunca con un $46.7 \%$, seguidamente la alternativa casi siempre con un $33.3 \%$, luego la alternativa algunas veces con resultados de $13.3 \%$ y por último la alternativa siempre con un $6.7 \%$.

Por otro lado, la programación de actividades, obtuvo como resultado una media de 2.13, la cual de acuerdo al baremo de interpretación la ubica en la categoría baja, resultado que refleja las demoras, desfases, poca definición y secuencia lógica de las actividades, que se presentan en la fase de ejecución de las obras de viviendas de interés social.

El resultado alcanzado se contrapone con lo planteado por, Guido y Clements, (2003) para quienes la programación de actividades es una relación de actividades específicas, detalladas, necesarias para realizar el proyecto global, en particular en proyectos pequeños. Sin embargo, para aquellos en los que se usa una estructura de división de trabajo, la persona o equipo responsable de cada tarea puede definir las actividades individuales. Una actividad es una pieza de trabajo que requiere de tiempo.

Como resumen, del análisis de la programación que se realiza en estos 
proyectos puede mencionarse que la ésta corresponde a un proceso sistemático en la cual se plasman las actividades de trabajos que incluye el proyecto $y$ al aplicarlo de manera eficiente se evitarían contratiempos durante la ejecución del proyecto.

En cuanto a la procura, el mayor porcentaje lo obtuvo la alternativa de respuesta nunca con un $40 \%$ seguido de la alternativa casi siempre con un $33.3 \%$, luego la alternativa algunas veces con un $26,7 \%$ y por último la alternativa siempre que alcanzó cero como resultado porcentual.

En relación a la media del proceso de procura que se realiza en estos proyectos se obtuvo un resultado de 2,07 , el cual al ser comparado con el baremo de interpretación lo ubica como bajo, es decir, muestra debilidades en la gestión de procura en su fase de ejecución, lo cual afecta el cumplimiento de metas propuestas.

Los resultados obtenidos contrastan con lo expuesto por Espinoza (2009), cuando menciona que hay que tener presente las instalaciones necesarias, el material los instrumentos, herramientas, equipos, entre otros. Es decir, todos aquellos materiales que sean necesarios para la ejecución del proyecto.

En este mismo orden, el análisis de los resultados obtenidos en el manejo de contrato, muestran que el mayor porcentaje lo obtuvo la alternativa de respuesta casi siempre con un $40 \%$, seguido de algunas veces con un $33.3 \%$, luego la alternativa siempre con un $20 \%$ y por último la alternativa nunca que obtuvo un $6.7 \%$.

Con respecto a la media de manejo de contrato, se obtuvo un resultado de 2.72 , valor que comparado con el baremo de interpretación lo ubica en la categoría de moderado, lo cual muestra en la fase de ejecución ciertas deficiencias en cuanto al manejo de contrato.
El resultado obtenido difiere de lo planteado por Guido y Clements, (2003) cuando definen el contrato como un vehículo para establecer buenas comunicaciones entre el cliente y el contratista y llegar a una comprensión mutua con claras expectativas que aseguran el éxito del proyecto.

De lo anteriormente expuesto se puede inferir que la relación contratista cliente en la que debe quedar claro cómo se manejará el contrato, se cumple con ciertas limitaciones.

Con respecto a la inspección de obra, los resultados muestran que el mayor porcentaje lo obtuvo la alternativa de respuesta nunca con un $40 \%$, seguido de casi siempre con un $33.3 \%$, luego la alternativa algunas veces con un $26.7 \%$ y por último la alternativa siempre que resultó con cero como valor porcentual.

En relación a la media del proceso de inspección de obra, este alcanzó como resultado un 2.07, el cual al ser comparado con el baremo de interpretación lo ubica en la categoría baja, lo que evidencia debilidades en la fase de ejecución en cuanto a inspección de obras, lo cual se traduce en falta de control y seguimiento de la obra, por baja presencia de personal de inspección en la misma.

Este resultado contrasta con lo planteado por Merrit, Loftin, Rickett (2004), quienes consideran que la inspección y consulta periódica durante la construcción, son normalmente parte de las responsabilidades del ingeniero inspector y que estas labores comprenden visitas periódicas al lugar de la construcción. La función del ingeniero del lugar de construcción, y del personal es inspeccionar que el trabajo se apegue a los requisitos del contrato, a los documentos y conceptos del diseño. 
La inspección de obra, expresan los autores, se refiere a la revisión periódica, hay que comprobar si todo realmente va según los cronogramas realizados.

Según los autores mencionados, la inspección de obra no solo es revisión que corresponde al ingeniero inspector, sino que ésta incluye inspecciones tipo visitas periódicas, no solo del ingeniero inspector, sino también del ingeniero constructor o líder del proyecto y del personal que de alguna manera deba llevar seguimiento y control de la obra.

En cuanto a la forma que se utiliza para el pago de los compromisos en estos proyectos el mayor porcentaje de su resultado lo obtuvo la alternativa de respuesta siempre con un $40 \%$, seguido de casi siempre con un $40 \%$, luego la alternativa algunas veces con un $13.3 \%$ y por último la alternativa nunca con un $6.7 \%$.

Asimismo, la media del proceso de pago obtuvo un resultado de 2.91 que al ser comparado con el baremo de interpretación lo ubica en la categoría de moderado, esto indica moderado cumplimiento en cuanto al manejo del proceso de pago, bien sea en verificación y control de facturas o verificación de valuaciones antes de su entrega al ente contratante, lo que trae como consecuencias retardos en los pagos, durante la fase de ejecución del proyecto, de las obligaciones contraídas.

El resultado obtenido contrasta con lo planteado por Guido Y Clements, (2003) al considerar el cumplimiento del contrato como un medio para establecer buenas comunicaciones entre el cliente $y$ el contratista lo que permite arribar a una comprensión mutua con claras expectativas que aseguran el éxito del proyecto.
Finalmente, se presenta el resultado del cierre de contrato cuyo mayor porcentaje lo obtuvo la alternativa de respuesta nunca con un $40 \%$, seguido de casi siempre con un $33.3 \%$, luego la alternativa algunas veces con un $26.7 \%$ y por último la alternativa siempre con resultado de cero como valor porcentual.

De igual manera, la media de cierre de contrato, alcanzó un resultado de 2.07 , el cual al ser comparado con el baremo de interpretación lo ubica como bajo, es decir, existe baja habilidad y/o destreza gerencial en los gerentes y líderes del proyecto para dar cumplimiento a las actividades involucradas en el cierre del contrato, bien sea por falta de comunicación, de planificación lo que no permite culminar en la fecha prevista en el contrato.

Este resultado difiere, con lo planteado por el PMBOK (2008), que expresa que el proceso de cierre de contrato respalda al cierre del proyecto, ya que incluye la verificación de que todo el trabajo y todos los productos entregables han sido aceptables.

En relación a los resultados globales de la ejecución de los proyectos de construcción con una media de 2,23, al ser comparada con el baremo de interpretación hace que se ubique en la categoría baja, lo que traduce una baja efectividad en el manejo gerencial de los diferentes aspectos que deben ser administrados durante la fase de ejecución de los proyectos.

Estos resultados permiten inferir la necesidad de que los líderes de proyectos de ingeniería sean objeto de diversas acciones y estrategias de formación sobre el manejo de herramientas gerenciales, toma de decisiones y dirección de equipos de trabajo; que les capacite para sobrellevar de manera eficiente los 
inconvenientes que se presentan durante la fase de ejecución de proyectos de viviendas de interés social.

\section{CONCLUSIONES}

Las empresas objeto de estudio, tienen un bajo cumplimiento con los diferentes aspectos administrativos de la fase de ejecución.

El estudio realizado arrojó resultados de baja definición en el alcance lo que permite inferir que esto influye cuando, frente a dificultades relacionadas con disponibilidad de recursos materiales, mano de obra y equipos, se toman decisiones de manera improvisada e informal, lo que trae como consecuencia incrementos de costos.

La programación de actividades es baja, prácticamente no se aplica y si se aplica es solo al principio del proyecto, olvidando luego la importancia del mismo, muchas veces los cronogramas y curvas de avances se realizan sólo para cumplir requisitos exigidos por el ente contratante.

La gestión de procura es baja, a pesar de que los gerentes realizan esfuerzos por obtener los materiales en sitio y verificar las cantidades, pero muchas veces por falta de planificación y por gerenciar de manera informal, olvidan lo referido a la disponibilidad de mano de obra; lo que trae como consecuencia la paralización o retraso de actividades así como incrementos de costos por correcciones fuera de tiempo.

Asimismo, la empresa hace un manejo moderado de sus contratos, sin embargo muchas veces deja de gestionar algún documento esencial, como un acta de inicio, por dar inicio a la fase de ejecución en forma apresurada para quedar bien con el ente contratante. De igual forma no llevan de manera secuencial la documentación relativa al manejo del contrato, lo que genera desorden en la gestión del proyecto.
En relación con la inspección de obra, esta es cumplida en forma baja, por los líderes quienes se encargan de velar que todo esté en orden al inicio de la obra, del control y verificación de los productos entregables, así como de verificar los rendimientos de las actividades planificadas.

En cuanto a las formas de pago, ésta es manejada por los líderes de proyecto en forma moderada, las facturas se verifican antes de entregarlas. Muchas veces no elaboran valuaciones y encargan de ello a su equipo de trabajo, presentan y verifican las facturas de pagos más no los lapsos de tiempo de entrega, lo que trae como consecuencia retrasos en los pagos que alargan los tiempos de entrega del proyecto u obra.

Igualmente el proceso de cierre de contrato, es manejado por los líderes de manera baja ya que ni los líderes de proyecto ni su equipo de trabajo documentan lo referente al contrato de obra, solo gestionan lo esencial para el cierre del contrato como son la acta de terminación, de recepción definitiva, liberación de fianzas, seguros, garantías mantenimiento y equipos; las valuaciones y mediciones finales solo las elaboran cuando el ente contratante las exige. De allí que al cerrar el contrato no se puede evaluar de manera eficiente, como fue el desempeño del proyecto, ni visualizar los errores cometidos, así como las lecciones aprendidas.

\section{REFERENCIAS}

Briceño, L. (2006). Administración y Dirección de Proyectos: un enfoque integrado. 2da Edición. Universidad de Chile

Cartay, I (2010). Planificación y control en la ejecución de Proyectos. Manual CIED. Maracaibo Venezuela 
Chamoun, J. (2007). Administración Profesional de Proyectos - La Guía, Editorial McGraw-Hill Interamericana. Primera Edición México

Espinoza, M (2012). Análisis de Gestión de Proyectos de Desarrollo Social. Editorial Universidad de México. México

Guido, J y Clemets, J (2003). Administración Exitosa de proyectos. 3era Edición. Editorial International J Thompson. México

Krygier, A (2010). Cultura Corporativa y Desarrollo. Editorial Krygier de Cohen Francis

Merrit, F., Loftin, M., y Kent, R. (2004). Manual Del Ingeniero Civil. 4ta edición. Editorial McGraw-Hill Companies, Inc., USA
Miranda, J (2005). Gestión de Proyectos. 4ta Edición. Editorial MM Editores Colombia

PMBOK (2003). Guía de los fundamentos para la dirección de proyectos.3ra. Edición. Editorial Project Management Institute, Inc. Pennsylvania EEUU

PMBOK (2008). Guía de los fundamentos para la dirección de proyectos.4ta. Edición. Editorial Project Management Institute, Inc. Pennsylvania EEUU

PMBOK (2013). Fundamentos de la Gestión de Proyectos PMBOK - 5ta. Edición en Español 2013; Pennsylvania, Estados Unidos

Rico, H. (2009). Gerencia de Proyectos. Editorial FEPADE Fundación Empresarial para el Desarrollo Educativo. Salvador 